\title{
Symmetry of black hole ejections in mergers of galaxies
}

\author{
P. Heinämäki ${ }^{1,2, \star}$ \\ 1 Tuorla Observatory, Väisäläntie 20, SF-21500, Piikkiö, Finland \\ 2 Tartu Observatory, 61602 Tõravere, Tartumaa, Estonia
}

Received 10 March 2000 / Accepted 7 March 2001

\begin{abstract}
The process of formation of four black hole systems in the center of a galaxy is simulated. Using an evolutionary model for the mergings of galaxies we determine the black hole masses and the merger times of their host galaxies. Mergers are presumed to lead to the formation of binary systems. Their evolution inside the host galaxy is followed until a collapse of the black hole binary happens or until a new merger with another galaxy occurs. In this way we include the possibility that the binaries collapse before a multiple black hole interaction is reached. When a four black hole system is formed, its evolution is calculated until black hole mergers or escapes occur. Ejection parameters such as the bending angle and the distance ratio in two-sided black hole ejections have been studied and compared with observations of two-sided radio sources. The simulations produce a good agreement with observations of double radio sources. The results also predict a so far unobserved class of radio sources which one should be able to find in a careful study of extended radio sources which are not classical doubles.
\end{abstract}

Key words. black hole physics - galaxies: kinematics and dynamics - galaxies: nuclei - methods: numerical radio continuum: galaxies

\section{Introduction}

Powerful FR II (Fanaroff \& Riley 1974) radiogalaxies represent a quite well defined class of AGN. These so called classical doubles have two symmetrically aligned radiocomponents which often contain a high-brightness "hot spot" on one or both sides of the optically identified host galaxy. Usually, a low luminosity tail connects the nucleus of the host galaxy and the extended radio lobes.

There are two main categories of theories which have been used to explain this phenomenon, namely the so called beam theory (Blandford \& Rees 1974) and the slingshot theory (Saslaw et al. 1974). The latter is investigated in this paper. It is based on the well known properties of the interaction of gravitating bodies. In analogy to the dynamics of gravitating bodies in celestial mechanics and in stellar dynamics, e.g. in the dynamics of small star clusters, the theory has been applied to interacting black holes in galactic nuclei. Even though the beam theory is widely used in explaining double radio sources at present, there is still a need to find alternative and supplementary models to the beam model and also to the so-called unified scheme (Barthel et al. 1989). The latter models have problems e.g. with the high velocities needed to explain the asymmetries

* e-mail: pekheina@oj287.astro.utu.fi in the double radio sources (Best et al. 1995), problems in explaining the very large angles between the parsec and kpc-scale jets (Appl et al. 1996) and the persistent collimation problem of the large scale jets (Begelman et al. 1980). A detailed comparison of unified beam models and the slingshot model has been carried out by Valtonen \& Heinämäki (2000).

According to the basic idea of the slingshot theory, black holes $(\mathrm{BH})$ initially come together in repeated galaxy mergers. Under favorable conditions, dynamical interactions of BHs leads to their ejections from the galaxy nucleus. Ejected BHs produce extended radio lobes by jets of relativistic particles spreading out along the rotation axes of the accretion disks. Black holes which fly through the galaxy leave radiative tracks (trails) in the surrounding medium, often with various complexities in their trajectories (Valtaoja \& Valtonen 1984; Valtaoja 1986; Valtonen \& Kotilainen 1989). The slingshot process produces one-sided, two-sided, and three-sided ejections which may be connected with corresponding morphologies in the radio galaxies.

The chaotic nature of the few body problem forces us to resort to numerical computer simulations and therefore only statisticalproperties of the system can be 
studied. Also, the lack of exact General Relativistic solutions of the few-body problem limits us to approximations to the real situation. In our calculations, gravitational radiation is assumed to affect only two bodies at any one time (which is actually quite likely, considering the nature of the few body evolution). This has been taken into account by adding a frictional force to the Newtonian force law. This force leads to a loss of energy which is consistent with the expression for the loss of energy due to gravitational radiation from a two body system as described by Peters \& Matthews (1963). The lack of knowledge of the detailed initial conditions in such a system, together with the fact that a chaotic system is sensitive to initial conditions, causes unavoidable inaccuracy in the results. However, we note that present simulations show that in some cases a few black hole system originating via galaxy mergers may produce ejections and escapes of the black holes which have properties observed in double radio sources.

In the current view, black holes are common in the nuclei of galaxies (Kormendy \& Richstone 1995 and references therein; Maggorrian 1998). The origin of multiple $\mathrm{BH}$ systems in the nuclei of galaxies is expected when black holes come together in a merger of two galaxies which contain initially one or two BHs each. Recent studies suggest that such BH systems have been also observed (e.g. Gaskell 1996). The mergers of the galaxies may also be related to the formation of certain kinds of galaxies. In suspected merger remnants, one finds peculiar optical features and unusually blue extra-nuclear colors (Toomre \& Toomre 1972; Schweizer 1982; Kormendy \& Djorgovski 1989; Barnes \& Hernquist 1992) and they have been connected with the formation of elliptical galaxies through mergers of spiral galaxies. Heckman et al. (1986), Smith \& Heckman (1989), Smith et al. (1990) and Hutchings (1987) found that $30-50 \%$ of nearby $(z<0.5)$ powerful FR II type radio galaxies show evidence of a recent merger. Furthermore, radio-loud AGN are always associated with elliptical galaxies, while the same is not true for radioquiet AGN (Wilson \& Colbert 1995). Binary and multiple black hole systems (introduced by Valtonen et al. 1974 and Begelman et al. 1980) may have a variety of applications in the field of astrophysics; activity and variability of the quasars (Lehto \& Valtonen 1996), bending of jets from active galaxies (Gaskell 1996), radio properties of the radio galaxies (Wilson \& Colbert 1995) and activity far from the center of the quasars (Makino 1997 and references there in). Following these lines we, introduce in this paper some further consequences of the binary black hole concept.

\section{Previous work}

The three and four black hole systems are dynamically very unstable and usually end up in highly energetic one-, two-, or three-sided ejections. Two-sided ejections take place most probably when masses of interacting BHs are relatively equal (Mikkola \& Valtonen 1990; Valtonen et al. 1994, hereafter Paper I). This requirement decreases the number of suitable mergers from the point of view of the formation of double radio sources at least in two ways: First, the fraction of relative equal mass $\mathrm{BHs}$ is naturally limited, since the $\mathrm{BH}$ masses in a galaxy nucleus may vary over a broad range. Secondly, only a minority of two-sided ejections can reach such a distance from the nucleus that they become observable as a double radio source. The latter point was studied in Paper I, where the ratios of different ejection types corresponding to the observed radio galaxies and quasars were studied and plausible biases in the used samples were taken into account. For example, it was thought that selection effects rule out from the comparison those cases in which the mass of the central $\mathrm{BH}$ is more than half of the total mass of the system. If the mass of the $\mathrm{BH}$ is proportional to the radio flux, these requirements may cut off cases where the overwhelming brightness of the central component hides the real morphology of the source. Another selection criterion rules out cases in which the mass ratio of the ejected BHs exceeds four. In the subsample which included these two observationally based selection criteria, the main disagreement with observations was found with the relatively large distance ratios $D_{\mathrm{r}}$, while the bending angles $\Phi$ agreed well. When the selection effects were not taken into account, the agreement was not so good. Surprisingly, observations agreed better with simulated distributions of $\Phi$ and $D_{\mathrm{r}}$ in the case of four $\mathrm{BH}$ systems than with three $\mathrm{BH}$ systems. Figure 1 shows schematically the central galaxy and extended radio lobes according to the slingshot theory. Figure 1 illustrates also the definitions of the parameters $\Phi, D_{\mathrm{r}}=D_{\max } / D_{\min }$ and flux the ratio $F_{\mathrm{r}}=F_{\max } / F_{\min }$.

In Paper I we calculated about 80000 different four $\mathrm{BH}$ cases divided in eight different sets of varying initial compactness and masses. The final configurations of the systems were classified in eight categories depending on the ejection type. Depending on initial values, about 30 to $50 \%$ ( $30 \%$ in the present study) of cases ended up with ejections of $\mathrm{BHs}$ exceeding the escape velocity of the galaxy. One-sided ejections were found to be the most common case while the two-sided ejections were the second most common type. About $1 \%$ to $10 \%$ of all ejections could be classified as double radio sources. Furthermore, we found that the typical age of visibility of an ejected $\mathrm{BH}$ is about $10^{8}$ years.

In this paper, we concentrate on two sided ejections which may be related to the FR II type radio galaxies. We associate FR II galaxies with the cases were two black holes are ejected and a single black hole or a binary BH stays in the nucleus of the galaxy. Using the computer codes from Paper I and Valtonen (1996, hereafter MV96) we study the formation of FRII radio galaxies as a process which starts from several galaxies merging together in succession and which continues through binary $\mathrm{BH}$ evolution and four BH dynamics to the ejections of two black holes out of the host galaxy. In this way, the present paper is a more complete treatment of the problem than Paper I, which started from rather arbitrarily chosen initial conditions. 


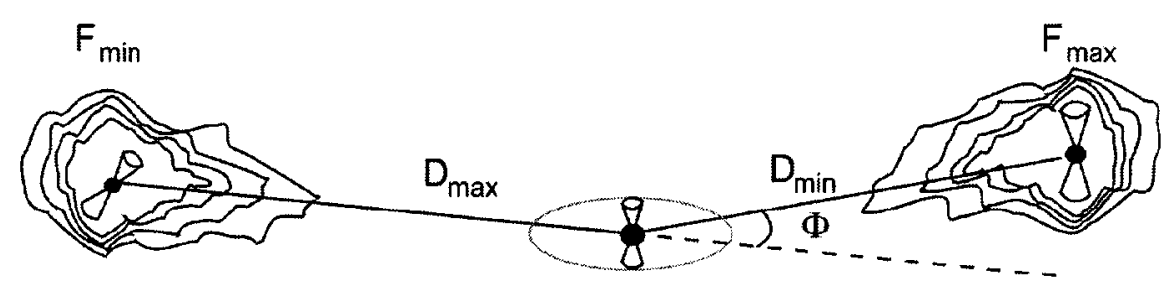

Fig. 1. A schematic diagram of the central galaxy and extended radio lobes. The definitions of the parameters are illustrated

\section{Calculations}

In Paper I we considered BH systems which started to interact in the nucleus of a galaxy when the BH masses, orbital orientations and the binary separations were randomly picked from a selected range. Here the calculations are initiated at an earlier stage of evolution of the four black hole formation.

The initial conditions are determined by the influence of the surrounding host galaxy. The general outline of the simulations may be divided in four stages: 1) two galaxies are presumed to have merged and the evolution of a binary $\mathrm{BH}$ system starts under the influence of the surrounding host galaxy, 2) two other galaxies are presumed to have merged and the evolution of the second binary $\mathrm{BH}$ system starts in the nucleus of the galaxy, 3) previous two galaxies with binary $\mathrm{BH}$ systems are presumed to have come together and the two binary $\mathrm{BH}$ systems start to approach each other in the nucleus of the galaxy and 4) the four BH system is formed and the dynamical evolution is followed until the slingshot process takes place.

On the way to the four $\mathrm{BH}$ system, the first requirement is that a galaxy with a BH binary system must have formed and it has survived until it meets the second binary $\mathrm{BH}$. We do not consider the possibility that a binary system is formed internally in the galaxy center. Thus, a key assumption is that our host galaxy has formed by galaxy successive mergers at some time intervals.

Lacey \& Cole (1993) derived an expression for the formation and the survival times of virialized halos (mainly dark) showing how the merger rate depends on the halo mass, the epoch, the initial spectrum of density fluctuations and the density parameter $\Omega_{0}$. They also calculated a mass accumulation history for a galaxy halo observed today using a probability distribution (Eq. (2.29) in Lacey \& Cole 1993)

$K(\Delta S, \Delta \omega) \mathrm{d} \Delta S=\frac{1}{(2 \pi)^{1 / 2}} \frac{\Delta \omega}{(\Delta S)^{3 / 2}} \mathrm{e}^{\frac{(\Delta \omega)^{2}}{2 \Delta S}} \mathrm{~d} \Delta S$,

where $K(\Delta S, \Delta \omega) \mathrm{d} \Delta S$ is probability of a change $\Delta S(\propto$ $\Delta M)$ in a step $\Delta \omega(\propto \Delta t)$. The former proportionality is based on Eq. (2.5) and latter one on Eq. (2.1) in Lacey $\&$ Cole (1993). Here $\Delta M$ is the halo mass added to the galaxy after the time step $\Delta t$. We create large numbers of different merger histories in accordance with Eq. (1). Examples of such histories are illustrated in Fig. 8 of Lacey \& Cole (1993). Thus we may say that our host galaxy has been put together from a large number of smaller galaxies; we do not know the exact history, but assume that all possible histories satisfy Eq. (1).

We take the four largest pieces from each merger history and say that these are the prime components out of which the host galaxy is made. Then we make the assumption that each of the four prime components has brought with it a $\mathrm{BH}$ into the host galaxy. The second major assumption is that the mass of the $\mathrm{BH}$ contributed by each prime component is directly proportional to the mass of the component itself. In this way we generate a spectrum of $\mathrm{BH}$ masses.

The order in which the BHs enter the host system comes from the same random process. At the first stage, two prime components merge, creating a binary $\mathrm{BH}$ at its center. At the second stage, another merger takes place creating the second $\mathrm{BH}$ binary. At the third stage the previous mergers combine to make the present day host galaxy, and the two BH binaries start their evolution toward, each other. During these stages two $\mathrm{BH}$ binaries evolve independently each inside its own galaxy. During the first two intervals the binary BHs may merge into a single black hole or they may survive. Similarly, the two $\mathrm{BH}$ binaries may come to interact with each other during stage three, or they may not. After these stages a four $\mathrm{BH}$ system may form and it may evolve until the third "probable" time limit is reached. At the same time the core radius, the mass and the density distribution of the galaxy also evolve according to MV96. For simplicity, only one type of host galaxy was considered at this phase of the calculation. The galaxy initially has total mass of $2.310^{11} M_{\odot}$, effective radius $5000 \mathrm{pc}$, a central velocity dispersion of stars $\delta v=220 \mathrm{~km} \mathrm{~s}^{-1}$ and the M/L-ratio 6.5 in solar units (Young 1976; Young et al. 1978). The initial semi-major axes $r$ of the binary $\mathrm{BH}$ orbits were taken to be $r=G\left(m_{1}+m_{2}\right) / \delta v^{-2}$ where $m_{1}$ and $m_{2}$ are the masses of the $\mathrm{BH}$ components. Also, the eccentricities $e$ of the binary $\mathrm{BH}$ systems were kept constant at $e=0.3$. The eccentricity seems to be rather unimportant to the overall binary evolution (Mikkola \& Valtonen 1990; Quinlan $\&$ Hernquist 1997). It was decided that a four BH system was created when the pericenter distance of the outer binary has become smaller than three times the semi-major axis of the inner binary. Here the inner binary means the wider of the two original binaries, and the outer binary refers to the relative orbit of the centers of mass of the 
two original binaries. In the case that the distance condition is not reached in a given time or when the original binary system collapsed we skipped to the next case. If two BHs come too close to each other, gravitational radiation forces the BHs together in a very short dynamical time scale (the planned space-based detector LISA may observe gravitational radiation pulses released in this final plunge of binary members, Haehnelt 1994). Thus it is necessary that the $\mathrm{BH}$ binary must survive in the conditions of the galaxy nucleus until it meets another galaxy with its binary BH system. This leads unavoidably to a decrease in the number of successful ejections, because only a minority of the cases end up in the four BH interaction. Anyway, we keep track of the "unsuccessful" cases for statistical purposes.

MV96 pointed out that there are at least three mechanisms which reduce the orbits of binary black holes in a galaxy center: 1) stars which flow past the binary. Stars gain kinetic energy and thus the binary shrinks; 2) gravitational radiation; and 3) cooling flow of gas which increases binary mass and may decrease the separation of binary members. There is only a finite reservoir of stars which can interact with the binary. Once the interacting stars have been ejected, a so-called loss cone forms. This means that in the stellar velocity distribution, those stars are missing whose velocity vectors lie in a cone which contains the binary and its near surroundings (Frank \& Rees 1976). The loss cone may be refilled if the stars in the nuclear region are sufficiently perturbed by external influences. In this work we have assumed that the loss cones are refilled, i.e. loss cones do not form. Because of this assumption, the orbital evolution of black hole binaries is faster than when loss cones do form. This assumption is not crucial since according to Rajagopal \& Romani (1995) the losscone effect does not affect the stability of a BH binary in Hubble time. On the other hand, Quinlan (1997) pointed out that the wandering of the binary from the galaxy center causes continued hardening of the black hole binary. In effect, the neglect of the loss cones in the present work amounts to accepting this wandering as the agent which constantly refills the loss cones. Thus the effect of the stars is maximized.

Items one and two follow the description of MV96 where (according to Mikkola \& Valtonen 1992) stars which pass close to the binary change the semimajor axis by

$\mathrm{d} a=a^{2} R_{a} \pi G \rho v^{-1}$.

Here $R_{a}=-6.5 /\left[1.0+2.44(v / W)^{2}\right]$ is the rate coefficient and $W$ is the rms relative speed of the binary members, $G$ is the gravitational constant, $a$ semimajor axis of the binary and $\rho$ is the density of stars which flow at the typical velocity $v$ at a large distance from the binary and past it. Valtonen (1996) found that if the mass of the stars which interact with the binary is lower than the binary mass, the binary does not collapse in the Hubble time. For example, in the case of a three billion solar mass binary system, the refilling of the loss cone should have occured about 200 times before enough orbital energy of the binary (initial radius $0.6 \mathrm{pc}$ ) has been carried away to cause the merger of the binary members. During the evolution, the initial density of stars within the core radius $r_{\mathrm{h}}=G(m+$ $M) v^{-2}$ (dynamically controlled by the binary) changed. This adjusted density distribution was taken into account during the calculations.

The change of $a$ by gravitational radiation can be written as

$\mathrm{d} a=-64 / 5 G^{3} m M(m+M) c^{-5} a^{-3} f(e)$,

where $m$ and $M$ are the masses of the binary members, $f(e)$ is a function of eccentricity. Using the distance unit $1 \mathrm{pc}$ and the mass unit $1.08310^{9} M_{\odot}$, the lifetime due to gravitational radiation of a circular binary of semimajor axis $a$ is $T_{\mathrm{c}}=4.610^{11} a^{4}[m M(m+M)]^{-1}$ years. Thus, to exceed the typical lifetime between mergers of galaxies (about $10^{9}$ years), the distance between binary members should be $a \geq 0.22[m M(m+M)]^{0.25}$ parsecs. If we assume that the galaxy harbours two billion solar mass black holes, their lifetime as a binary will exceed the Hubble time if the distance between the binary members is about $0.3 \mathrm{pc}$ or greater.

According to Roos (1988) the interaction of the binary with another supermassive black hole which spirals inward changes momenta of stars in the main galaxy and fills the loss cone of the initial binary. If the approach of the third black hole takes long enough, this may lead to the collapse of the binary before the actual few body interaction starts. However, such a long and protracted approach is very unlikely to occur in nature (Valtonen et al. 1994). A better way to view this situation is to regard the binary and the third body as forming a wide binary (Valtonen 1994, 1996). The wide binary has a loss cone more massive than the loss cone of the inner binary. This leads to a faster decrease of the orbital radius of the wide binary than of the inner binary and finally few body evolution begins.

The increase of binary mass by the cooling flow of gas has been added to the simulations using the results from the simulations by Bate (1997) and Bate \& Bonnell (1997). They studied accretion during the binary star formation using ballistic and gaseous models. Qualitatively both models gave the same results. The most important parameters in the evolution of the binary component masses and of the binary component separation were the angular momentum of the accreted gas relative to the angular momentum of the binary and the initial mass ratio of the binary members. However, the eccentricity of the binary system was not found to be important. Their main results were as follows: In the case of accretion of gas with low angular momentum, the primary body increases its mass more than the lighter one. In the case of high angular momentum, the process is reversed. Furthermore, separation of the primary members decreases when the infalling gas has low angular momentum and increases in the high angular momentum case. Recent studies of the evolution of accreting binary systems by Bate (2000) confirm the previous picture. However, it was proposed that the effect of 
increasing the binary separation is probably smaller than in Bate (1997) and Bate \& Bonnell (1997) due to the interaction of the binary with the circumbinary disc. In the case of high angular momentum of the infalling gas, the evolution of the binary separation and the formation a circumbinary disc depend on the properties of the infalling gas while the mass ratio of the binary has almost no effect. Since our BH binaries are very compact $(\sim 1$ parsec) in the galactic scale we may approximate the gas infall with the high angular momentum case relative to the binary system. The BHs own spin angular momentum can be neglected.

In the case of high angular momentum, the change in the mass ratio $q$ will depend on the mass increase $\Delta M$ approximately as follows Bate (2000):

In the case that $q<0.67$

$\Delta q=0.25 \times \frac{\Delta M}{M}$

and in the case that $q>0.67$

$\Delta q=0.75 \times(1-q) \frac{\Delta M}{M}$

where $M$ is total mass of the binary system.

Following MV96 we have assumed that one half of the gas comes from direct parabolic orbits to the binary and rest from random orbits. Thus, half of the gas accretion will take place from a circumbinary disk, the initial orientation of which is derived from the galaxies which have merged.

The average rate of change of separation $a$ may be written

$\Delta a=0.375 \times a \frac{\Delta M}{M}$.

When the second galaxy with a BH binary enters the system, the mass flow is divided first between the two binaries as was done with the single binary and after that, the gas is further divided between the BHs in each system. In our calculations, the rate of cooling mass flow was assumed to be $0.2 M_{\odot} / \mathrm{yr}$.

\section{Results}

The initial number of the four galaxy systems which made up the host galaxy sample was 10000 . BH masses in the range $0.1-1.010^{9} M_{\odot}$ were picked from the probability distribution of Eq. (1), to be placed in the galaxy centers.

During the evolution which leads to the formation of a four BH system in the galaxy center, two thirds of the cases were "unsuccessful". These missed cases were the results of binary $\mathrm{BH}$ mergers before a new galaxy was encountered within the given time limits. Finally, we had 3156 "successful" cases with four BH systems. Their evolutions were calculated until escapes or mergers took place or the time limit was reached. The number of the "successful" cases depends on the rate of the cooling mass flow. Varying the values from $0.05 M_{\odot} / \mathrm{yr}, 0.2 M_{\odot} /$ yr to
Table 1. The number of end results in different ejection categories when the previously unfinished cases are repeated with a smaller pericenter distance by a factor of $f$ relative to the original value

\begin{tabular}{lcccccccccc}
\hline$f$ & 1.0 & 0.8 & 0.7 & 0.6 & 0.5 & 0.4 & 0.3 & 0.2 & 0.1 & Total \\
\hline 1-sided & 890 & 373 & 225 & 138 & 125 & 74 & 41 & 26 & 11 & 1905 \\
2-sided & 532 & 109 & 88 & 27 & 40 & 17 & 15 & 6 & 4 & 836 \\
3-sided & 291 & 36 & 24 & 4 & 3 & 3 & 3 & 2 & 2 & 369 \\
unfinished & 1441 & 923 & 586 & 417 & 249 & 156 & 97 & 63 & 46 & 46 \\
\hline
\end{tabular}

$1.0 M_{\odot} /$ yr for the cooling mass flow, we found that corresponding numbers of the "successful" cases were 2891, 3156 and 4954. These numbers show that results in such calculations cannot be very robust as long as better observational estimations are not available for the essential parameters. Moreover, we found that if the merging timescale is decreased by a factor of two, the number of unsuccessful cases decreases by the same factor. Thus the merging timescale between galaxies seems to determine the effectiviness of the slingshot process.

Final configurations were classified in four categories: 1-sided, 2-sided, 3-sided and unfinished. In spite of relatively long integration times (10 crossing times of the system), about half of all cases fell in the category "unfinished". Typically these cases are very stable configurations where the two binaries have orbital angular momenta with opposite signs. The integrations of these cases were restarted with the same initial parameters except that the pericenter distances were decreased by a factor of $f$ from the original value. The idea was to accelerate the binary BH system evolution which is assumed to be under the influence of dynamical friction caused by stars in the galaxy center. This was repeated eight times and every time the pericenter distance was made smaller than previously. Table 1 summarizes the results at each round. It shows that the evolution of 10000 four galaxy systems leads finally to 836 two-sided ejections. The decreasing of the pericenter distance did not change the distributions of the parameters studied.

The results of the two-sided escapes from the galaxy are compared with the catalog of two-sided radio galaxies and quasars by Nilsson (1998). The catalog contains 1038 sources which include 725 objects collected from Herbig \& Readhead (1992) (which is a 2.5 Jy sample) and 313 sources from various other samples. The main criterion to be included in this sample was that the sources must represent a clear FR II morphology. This work has used all the sources (368 objects) from the catalog for which the distance ratio $D_{\mathrm{r}}$, the flux ratio $F_{\mathrm{r}}=F_{\max } / F_{\min }$, the projected linear size and the bending angle $\Phi$ have been available.

In Paper I we incorporated two selection effects in our simulations to simulate obvious biases in observations: the central $\mathrm{BH}$ mass ratio to the total $\mathrm{BH}$ mass should be less than 0.5 and mass ratio of the ejected $\mathrm{BHs}$ should not exceed 4 . The former rule (which seems to be more or less 




Fig. 2. The curves show the evolution of the radio power $P$ as a function of the linear size $D$ at different initial velocities $v_{0}$ for the ejecta according to the model by Valtaoja \& Valtonen (1984). Black circles represent galaxies and white ones quasars from the catalog of double radio sources by Nilsson (1998). The scale of Valtaoja \& Valtonen (1984) has been increased by a factor of 4 to account for different values of the Hubble constant and for the two-sidedness of the radio sources

the selection rule in observations if radio flux scales as $\mathrm{BH}$ mass; only $7 \%$ of the sources in the catalog by Nilsson exceed this limit) is still used in the present study but the latter one is replaced by a quantitative model of lobe brightness and probability of inclusion in flux-limited samples. The model used is the model by Valtaoja \& Valtonen (1984). They showed that the radio luminosity depends strongly on the initial velocity of the ejected BHs as well as on the distance from the center of the galaxy.

The model is summarized in Fig. 2 where different curves represent different initial velocities for the ejected black holes, in a plot of radio power $P$ as a function of linear size. In addition, the observations by Nilsson (1998) are added into the luminosity-size diagram of Fig. 2. Black circles represent galaxies and white ones are quasars.

The upper envelope of the model curves by Valtaoja \& Valtonen (1984) outlines the overall area covered by the observed double radio sources. It is probable that the lack of quasars at low $P$ values is a selection effect. An analytical model was used to describe the escape orbits in Fig. 2.

The radio luminosity $P$ depends on the $\mathrm{BH}$ velocity $v$ relative to the escape velocity from the galaxy $\left(v_{\text {esc }}\right)$ and on the time since escape ( $T$, units of a million years), as follows $\left(T \leq 60\right.$ and $\left.v_{0}=v / v_{\text {esc }} \leq 1.16\right)$

$\log P=9.5\left(1-{\frac{(T-33)^{2}}{85}}^{2}\right)+(-0.8+0.0052 T)\left(\log v_{0}+1\right)$. $(7)$
In the case that $T \geq 60$ and $v_{0} \leq 1.16$

$\log P=3.6+\frac{(6.5-0.0325 T)}{3.5}\left(\log v_{0}+4.5\right)$.

When $v_{0} \geq 1.16$ and $10 \leq T \leq 200$ we used

$\log P=5.53-(1.16-0.003 T)\left(\log v_{0}-1.53\right)$.

Distance to the outer edge of each radio component was determined by

$D=3.11 T \sqrt{v_{0}^{2}-0.89^{2}}$.

Finally, we weighted the numbers by

$p=\left(P_{1}+P_{2}\right)^{1.5}\left(e^{-t / t_{1 \mathrm{im}}}\right)^{1.5}$,

where $p$ is the weight factor, $t$ is the time of observation (sampled at equal intervals of $10^{7}$ years up to $210^{8}$ year) and $t_{\text {lim }}=10^{8}$ years which corresponds to the average lifetime of the radio sources (Paper I). Thus the exponential part imitates the exponential decrease (decay) of the mass of the accretion disk (the fuel of the black hole) when time passes. The factor $p$, probability that a certain radio source will enter the flux limited source sample, is constructed assuming that the radio luminosity of the source depends on the sum of the component luminosities $P_{1}+P_{2}$ (calculated from Eqs. (5)-(7)). The exponent 1.5 in each factor comes from simple assumption that the number $N \propto R^{3}$ and the power $P \propto R^{2}$, where $N$ is the number of sources observed out to the distance $R$ and $P$ is the luminosity of the the faintest observable source at distance $R$.

Using this analytical model for the curves shown in Fig. 2, we calculated flux-weighted histograms for the distribution of the simulated radio sources. The results are plotted in Figs. 3-5 and 8. Figure 3 shows the probability $p$ in the flux ratio $\left(F_{\mathrm{r}}\right)$ - distance ratio $\left(D_{\mathrm{r}}\right)$ plane. There are two separate groups of sources. One group is symmetric with $F_{\mathrm{r}}$ and $D_{\mathrm{r}}$ values close to unity and the other group includes asymmetric sources whose flux ratios are of the order of 100 . We propose that the latter group of sources is easily missed in the radio galaxy samples. Instead, the former ones satisfy the definition of classical doubles, and are thus included in the samples of double radio sources.

The range of flux ratios for classical doubles appears to be about $-1<\log F_{\mathrm{r}}<1$. We adopt this range as our selection criterion for a source to be classified as a double radio source and use it when we compare simulations with the observations.

Figure 4 (solid dots) shows the distribution of the symmetry parameter $\log D_{\mathrm{r}}$ in the weighted simulations using Eq. (8) and covering the doubles for which $-1<\log F_{\mathrm{r}}<$ 1. Open circles show the corresponding distribution for the range $\left|\log F_{\mathrm{r}}\right| \geq 1$. The latter distribution is rather uniform and contains sources which have large asymmetries. For comparison, triangles illustrate the observed distribution of the distance ratio $D_{\mathrm{r}}$. This distribution is very similar to the distribution with solid dots. Thus the discrepancy found in Paper I between theory and observations has disappeared in the present study. 


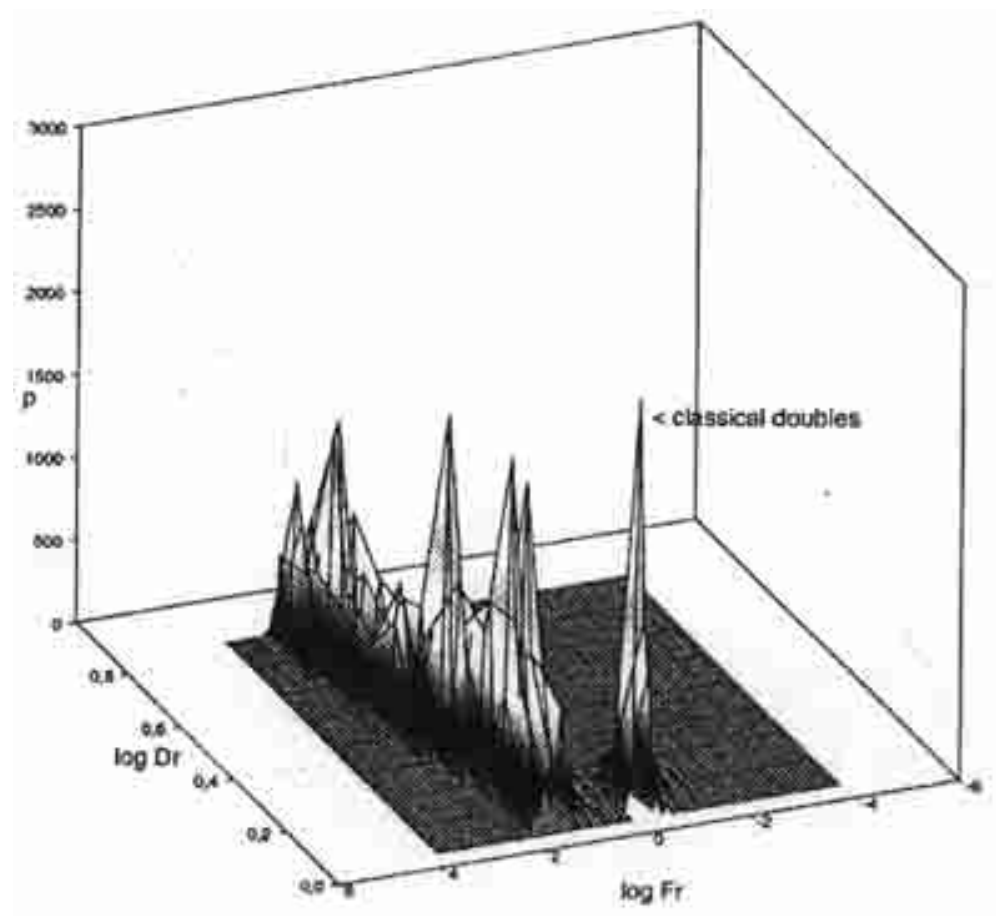

Fig. 3. The flux-weighted probability distribution of simulated double radio sources in the distance ratio $D_{\mathrm{r}}-$ flux-ratio $F_{\mathrm{r}}$ plane, for subsample $M_{\mathrm{c}} / M_{\mathrm{tot}}<0.5$. Two separate groups stand out; the peak of classical doubles $\left(\left|\log F_{\mathrm{r}}\right|<1\right)$ and "mountain range" of strongly asymmetric doubles $\left(\left|\log F_{\mathrm{r}}\right| \geq 1\right)$

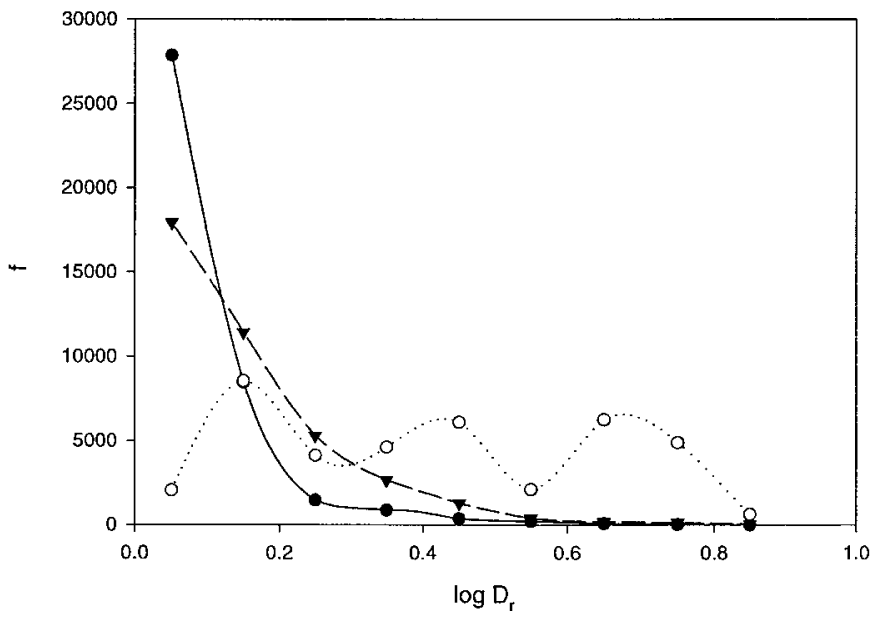

Fig. 4. The flux-weighted probability distribution of the distance ratio $\log D_{\mathrm{r}}$ for the simulated double radio sources with $\left|\log F_{\mathrm{r}}\right|<1$ (solid dots). Same as previous but with $\left|\log F_{\mathrm{r}}\right| \geq 1$ (open circles) and the observed distribution of $D_{\mathrm{r}}$ from Nilsson (1998) (triangles)

Figure 5 (solid dots) shows the probability distribution for the bending angle $\Phi$. Bending angles do not depend very much on the flux ratio. Thus we show all bending angles within the range $-4<\log F_{\mathrm{r}}<4$. The distribution is rather similar to the distribution of open circles, which illustrates the corresponding observations. Simulations also produced some very large bending angles (more than $90^{\circ}$ ) which are not seen in the Nilsson (1998) sample. Probably sources where the bending angles are more than 90 degrees

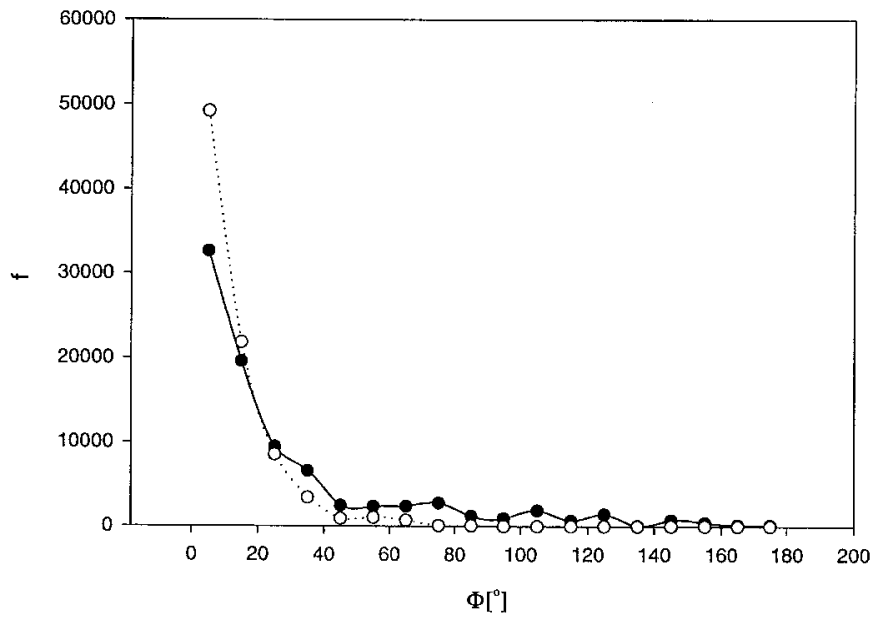

Fig. 5. The flux-weighted probability distribution of the bending angle $\Phi$ for all two-sided ejections where $M_{\mathrm{c}} / M_{\text {tot }}<0.5$ (solid dots) and the observed distribution of the bending angle $\Phi$ from Nilsson (1998) (circles)

(and thus the two radio lobes are on the same side of the parent galaxy) are not so easily classified as double radio sources.

According to the slingshot theory, the radio power of the radio lobes depends on the $\mathrm{BH}$ mass and the amount of "fuel" existing in its accretion disk (Rees \& Saslaw 1975). The latter point may be connected to the past evolution of the BHs inside the nucleus of the galaxy and to the distance between the BHs at their closest encounters with 


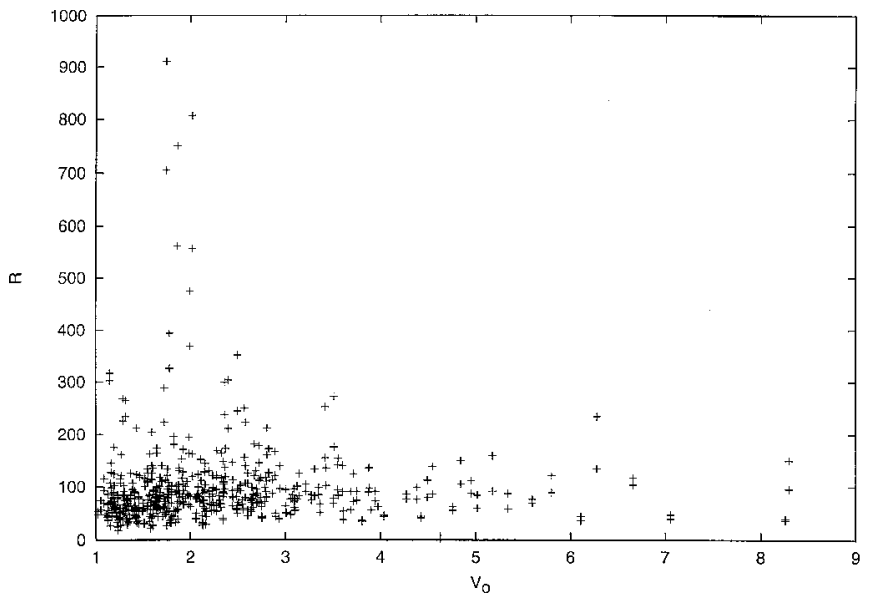

Fig. 6. The truncation radius of the accretion disk $R$ in units of the Schwarzschild radius of the ejected black hole as a function of the average velocity of the ejected bodies $v_{0}$. The sample with $M_{\mathrm{c}} / M_{\text {tot }}<0.5$

each other before the escapes occurred. It is obvious that these close interactions truncate the accretion disks of the ejected BHs which will reduce its brightness (Lin \& Saslaw 1977). To gain enough energy for escape, BHs should approach each other rather closely. We used the Roche limit condition to determine the survival radius of the accretion disks of the passing BHs. The quantity $R$, in units of the Schwarzschild radius of the escaping BH, in Fig. 6 indicates what size accretion disks survive the close passages in each ejection as a function of the average speed of the escaped bodies. Figure shows that typically $R \sim 70$ and almost always $25<R<250$.

So far we have discussed cases where the central mass condition $M_{\mathrm{c}} / M_{\text {tot }}<0.5$ is satisfied. Here $M_{\text {tot }}$ is the total $\mathrm{BH}$ mass and $M_{\mathrm{c}}$ is the total $\mathrm{BH}$ mass remaining at the center of the galaxy after the ejections. Figure 7 shows the $R-v_{0}$ plot for $M_{\mathrm{c}} / M_{\mathrm{tot}} \geq 0.5$. Comparing Figs. 6 and 7 we calculate that rather large accretion disks can survive when $M_{\mathrm{c}} / M_{\text {tot }}>0.5$. Then quite commonly $250<R<2500$ and $1 \leq V_{0} \leq 2$ i.e. BHs with relatively large accretion disks escape with a low velocity. The two escapers leave the galaxy in two independent processes, the result of which is that the $\Phi$-angle is totally random. Only by accident, when the $\Phi$-angle happens to be small, would a pair of ejections like this be classified as a classical double source. It is interesting to note in this connection that in a sample of core-dominated radio sources by Murphy et al. (1993) there are 25 2-sided sources, for which the median value of $\Phi=25^{\circ}$, much greater than the typical median value $\Phi=10^{\circ}$ for classical double radio sources. Their median linear size is also only about one half of the corresponding value for the classical doubles.

According to the standard theory of the Keplerian accretion disk (Shakura \& Sunyaev 1973; Novikov \& Thorne 1973; Shapiro \& Teukolsky 1983) the disk can be divided into three regions as a function of $r$ (distance from the $\mathrm{BH})$; an outer, a middle and an inner region. Most of the

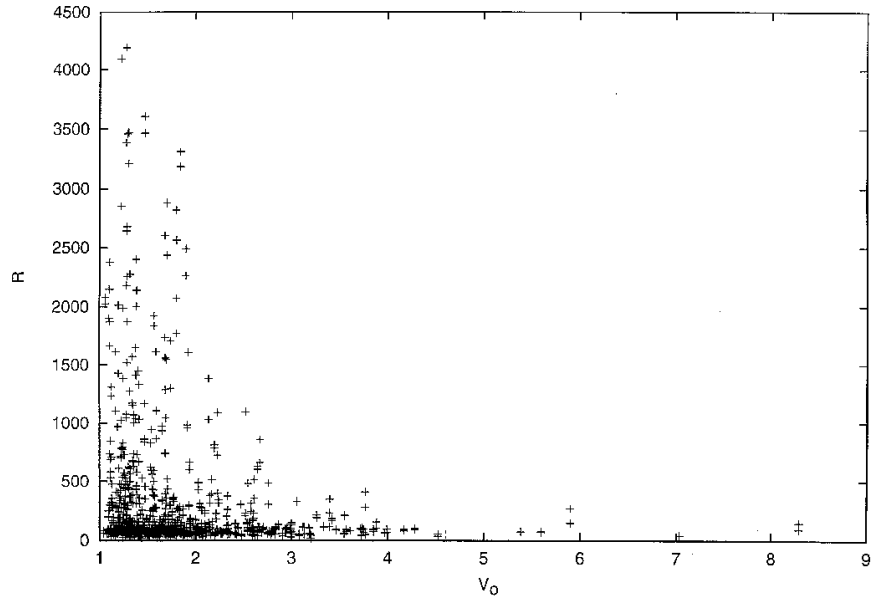

Fig. 7. The truncation radius of the accretion disk $R$ in units of the Schwarzschild radius of the ejected $\mathrm{BH}$ as a function of the average velocity $v_{0}$ of the ejected bodies. The sample with $M_{\mathrm{c}} / M_{\text {tot }}>0.5$

radiation comes from the inner region. It is probable that close encounters with a passing $\mathrm{BH}$ will sweep away the outer parts of the accretion disks.

Let us calculate the radius and the mass of the inner region with typical parameter values. According to the Keplerian accretion disk models the, outer radius $r_{\mathrm{i}}$ of the inner region is determined by

$\frac{r_{\mathrm{i}}}{r_{\mathrm{schw}}}=4.110^{8} \alpha^{2 / 21}\left[\frac{M_{\mathrm{BH}}}{M_{\odot}}\right]^{-2 / 3} \dot{M}^{16 / 21}$.

The unit of $\dot{M}$ is $M_{\odot} /$ yr. Substituting viscosity parameter $\alpha=10^{-4}, M_{\mathrm{BH}}=410^{8} M_{\odot}$ and accretion rate $\dot{M}=0.1 M_{\odot} /$ yr in the previous equation yields the radius $r_{\mathrm{i}} \sim 55 r_{\mathrm{schw}}$ (where $r_{\text {schw }}$ means Schwarzschild units). Naturally, the solution depends on the poorly-known values of $\alpha$ and $\dot{M}$. Using previous units and the surface density $\Sigma$ of the disk by Shapiro \& Teukolsky (1983)

$\Sigma=8.110^{-14} M_{\mathrm{BH}} \alpha^{-1} \dot{M}^{-1}\left[\frac{r_{\mathrm{i}}}{r_{\mathrm{schw}}}\right]^{7 / 2}$,

where $M_{\mathrm{BH}}$ is in units of $M_{\odot}, \dot{M}$ in units of $M_{\odot} / \mathrm{yr}$ and $\Sigma$ is in units of $M_{\odot} / r_{\mathrm{i}}^{2}$.

We can estimate the integrated mass of the inner region of the accretion disk

$M_{\mathrm{acc}}=2 \pi \Sigma \int_{0}^{1} R_{\mathrm{d}}^{5 / 2} \mathrm{~d} R_{\mathrm{d}}$,

where $R_{\mathrm{d}}=r / r_{\mathrm{i}}$. Even though the previous equation applies only up to the outer radius of the inner region of the disk, we may extrapolate it also somewhat further out. Thus we find that within the typical accretion disk cutoff radius $R=r / r_{\mathrm{schw}}=70$, the mass of the accretion disk is $\sim 10^{7} M_{\odot}$. This means that with assumed constant accretion rate the ejected $\mathrm{BH}$ will radiate about $10^{8}$ years. Hence we conclude that the previously-used value $t_{\lim }=10^{8} \mathrm{yr}$ is appropriate. It must be reminded 


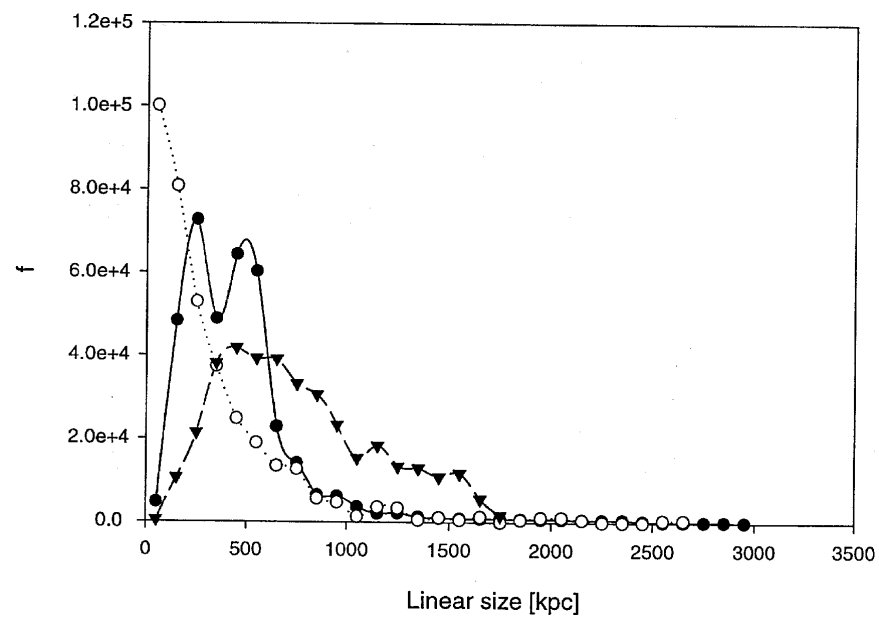

Fig. 8. The flux-weighted probability distribution of the linear sizes for the sample $\left|\log F_{\mathrm{r}}\right|<1$ and $M_{\mathrm{c}} / M_{\text {tot }}<0.5$ (solid dots). The distribution has been corrected for random projections and for a distribution of host galaxy scale sizes. As for the previous but for $\left|\log F_{\mathrm{r}}\right| \geq 1$ (triangles) and the observed distribution of double radio sources from Nilsson (1998) (open circles)

that the result depends on the model and especially on the viscosity parameter, which is poorly known. Thus the uncertainty of the result is propably a factor of 100 . Using these values, the radiation lifetime agrees with the estimations of the ages of double radio sources (Wilson \& Colbert 1995; McCarthy et al. 1977 and references therein; Zirbel 1997).

In Fig. 8 we show the linear size distributions for the simulations in the two separate groups corresponding to Fig. 4. As described previously in this paper, the sources are sampled at equal time intervals and at each time the weight factor of Eq. (9) is calculated. The distribution represents the sum of the weight factors at each distance $D_{\max }+D_{\min }$ from the center of the galaxy.

In addition to the factor $\left(P_{1}+P_{2}\right)$ in the weight factor p of Eq. (9) we have multiplied by the accretion disk lifetime factor $\mathrm{e}^{-t / t_{\mathrm{lim}}}$, where $t_{\text {lim }}$ is now the average time scale of decay of the accretion disk in the two components. It is calculated from equation $t_{\text {lim }}=\left(R / 70 r_{\text {schw }}\right)^{3.5} 10^{8} \mathrm{yr}$, where $R$ is the outer radius of the accretion disk. In our analytical model (Eqs. (5)-(8)), the model galaxy is M87, where luminosity is higher than the median value for the FRII radio galaxies in general (Zirbel 1996). To correct for this, the linear size distributions were constructed using the absolute magnitude distribution from Zirbel (1996, Fig. 6) together with the scaling laws by Young (1976). Also, random projection factors were included.

Figure 8 shows that the median value of the linear size in the slingshot model (solid dots) is about $400 \mathrm{kpc}$ while for observations it is about $200 \mathrm{kpc}$ (open circles). One reason for the discrepancy could be that have we overestimated the ages of the radio sources, by a factor of two. On the other hand, there is also a difference in the shapes of these two distributions. This is at least partly the result of not having non-escapers in our model. There are many BHs which do not quite reach the escape velocity. For example, the evolutionary curves between $v_{0}=0.8$ and $v_{0}=1.0$ in Fig. 2 contribute radio sources in the classical double radio source range, and they appear as FRII sources up to the peak radio luminosity and even a little beyond that point. Our model includes only the escaping BHs, $v_{0} \geq 1.0$, and therefore we expect a deficiency of small linear sizes in observations (open circles). This may explain the difference between the median sizes in the theoretical and observed radio source samples. Quantitatively, we know from the steep distribution of ejection velocities (Paper I) that the numbers of ejections in the range $0.8 \leq v_{0} \leq 1$ and in the range $v_{0}>1$ are comparable to each other. Since the former contribute mainly to the linear sizes below $200 \mathrm{kpc}$ and the latter above $200 \mathrm{kpc}$, it is likely that the median will be shifted close to this value when non-escaping $\mathrm{BHs}$ are included.

The present results suggest that the double radio sources with normal bending angles, but relatively large distance ratios and high $F_{\mathrm{r}}$, may exist. Sources like these are easily misclassified as 1-sided sources and left out from the classical 2 -sided radio source samples. Their average sizes are about twice as large as the sizes of classical double sources (Fig. 8, triangles).

\section{Discussion}

According to studies by Auriemma et al. (1977) and Woltjer (1990) the fraction of powerful radio galaxies among elliptical galaxies can be estimated at $\sim 10^{-4}$. Now it is possible to make a rough comparison between simulations and this value. Using the hierarchical halo merger model of Lacey \& Cole (1993), we simulated the accumulation of black holes in 10000 systems. We assumed that the masses of the BHs correlate with the masses of their host galaxies. We also require that the galaxy masses do not differ by more than one order of magnitude from each other since we want $\mathrm{BH}$ systems of relatively equal masses. This requirement decreased the number of cases to 6975 . After the merger process, 3156 galaxies contain a four BH system for further calculations. After the slingshot process, 836 cases ended up being two-sided. Finally, when the selection effect $M_{\mathrm{c}} / M_{\text {tot }}<0.5$ was introduced, 214 cases were left in the sample. But this sample still did not entirely agree with observations. If we neglect the asymmetric part of the population ending up in the "mountain range" $\log F_{\mathrm{r}} \sim-2$ in Fig. 3, and consider only the classical doubles peak, the final number of cases is about 40. Thus the ratio of simulated two-sided sources, which should be easy to observe, to the initial population of the model galaxies is about $40 / 10000=410^{-3}$. Thus, considering the typical lifetime of powerful radio galaxies in our model of $\sim 10^{8}$ yr relative to the age of the universe $\sim 10^{10} \mathrm{yr}$, the fraction of two-sided sources at the present time should be about $410^{-3} 10^{8} \mathrm{yr} / 10^{10} \mathrm{yr}=410^{-5}$. When we add to this a number of one-sided sources (some 
of which come from the "mountain range" of Fig. 3) and core-dominated sources, we are likely to end up close to the observed fraction of strong radio sources among elliptical galaxies.

Finally, it is worth mentioning an interesting scenario which arises from the VLBI observations by Readhead et al. (1996). They noted that about $10 \%$ of the powerful extragalactic radio sources should be classified as compact symmetric objects (MSO) indicating subkiloparsec scale high-luminosity lobes aligned symmetrically on both sides of the galaxy core. All MSO parent objects are elliptical galaxies. They propose a hypothesis in which MSOs evolve into large Fanaroff-Riley II objects. From the point of view of the slingshot theory, some of these objects may be explained by symmetrically ejected black holes from the galaxy nucleus. Depending on the height of the potential well of the parent galaxy, the ejected BHs may escape or turn and rush back towards galaxy nucleus. This stage of evolution (namely interplay) can repeat itself until mergers or escapes take place. Another possibility is that subkiloparsec scale jets are generated by the central $\mathrm{BH}$. If the parsec scale radio lobes are generated by the jets of the central $\mathrm{BH}$, then the projected angles between the jet and the ejection trails should generally differ from each other. This has been found e.g. by Appl et al. (1996) who notice that in about $50 \%$ of the cases the small scale jets are ligned up roughly perpendicular to the outer radio structure.

Results in this paper propose that the dynamical processes starting from galaxy mergers and from the formation of few body systems may finally end up in ejections, mergers and escapes of the black holes. The fraction of the two-sided sources produced by this evolutionary model agrees with the estimates of the space density of FRII radio sources. The model also predicts the existence of radio sources, for which the distributions are different from the classical double radio sources. These are the sources in the "mountain range" of Fig. 3 where the axial ratio $D_{\mathrm{r}}$ is high and the component flux ratio $F_{\mathrm{r}} \sim 10^{-2}$. Finding this radio source population is an observational challenge. Moreover, our simulations predict that there should also be a population of independently-ejected low mass BHs which typically move out at low speed but radiate for a long time due to their extensive accretion disks. They should show up as a weak radio source excess around radio galaxies. Some of them may even be seen optically if the accretion disk is at the upper end of the possible size range. But typically the accretion disks of ejected $\mathrm{BHs}$ are truncated, such that only the X-ray emitting central parts survive. Thus the ejected black holes may be best observed in X-rays and also in radio at the end points of the jets where the jet flow mixes with the interstellar medium (Valtonen 1999). These points are generally quite far (several kiloparsecs) from the BH itself.

The level of truncation of the accretion disks of the ejected BHs does not to correlate with the symmetry properties of classical double ejections. However, the estimated visibility lifetime of the ejected $\mathrm{BH}$ and the mean lifetime of the FRII radio sources determined from the linear size distribution are of the same order of magnitude. In this sense the truncation is an important process which determines the maximum size of a classical double radio source.

Acknowledgements. The author is grateful to M. Valtonen, L. Takalo and K. Nilsson for valuable discussions, comments and help. This study was partly supported by Academy of Finland (grant 46733).

\section{References}

Appl, S., Sol, H., \& Vicente, L. 1996, A\&A, 310, 419

Auriemma, C., Perola, G. C., Ekers, R., et al. 1977, A\&A, 57,41

Barnes, J. E., \& Hernquist, L. E. 1992, ARA\&A, 30, 705

Barthel, W. C., Hooimeyer, J. R., Schilizzi, R. T., Miley, G. K., \& Preuss, E. 1989, ApJ, 336, 601

Bate, M. R. 2000, MNRAS, 314, 33

Bate, M. R. 1997, MNRAS, 285, 16

Bate, M. R., \& Bonnell, I. A. 1997, MNRAS, 285, 33

Begelman, M. C., Blandford, R. D., \& Rees, M. J. 1980, Rev. Mod. Phys., 56, 255

Best, P. N., Bailer, D. M., Longair, M. S., \& Riley, J. M. 1995, MNRAS, 275, 1171

Blandford, R. D., \& Rees, M. J. 1974, MNRAS, 169, 395

Fanaroff, B. L., \& Riley, J. M. 1974, MNRAS, 176, 633

Frank, J., \& Rees, M. J. 1976, MNRAS, 176, 633

Gaskell, C. M. 1996, ApJ, 464, L107

Haehnelt, M. G. 1994, MNRAS, 269, 199

Heckman, T. M., Smith, E. P., Baum, S. A., et al. 1986, ApJ, 311,526

Herbig, T., \& Readhead, A. C. S. 1992, ApJS, 81, 83

Hutchings, J. B. 1987, ApJ, 320, 122

Kormendy, J. 1995, ARA\&A, 33, 581

Kormendy, J., \& Djorgovski, S. 1989, ARA\&A, 27, 235

Lacey, C., \& Cole, S. 1993, MNRAS, 262, 627

Lehto, H. J., \& Valtonen, M. J. 1996, ApJ, 460, 207

Lin, D. N. C., \& Saslaw, W. C. 1977, ApJ, 217, 958

McCarthy, P. J., Wil van Breugel, \& Spinrad, H. 1987, ApJ, 321, L29

Magorrian, J., Tremaine, S., Richstone, D., et al. 1998, AJ, 115,2285

Makino, J. 1997, ApJ, 478, 58

Mikkola, S., \& Valtonen, M. J. 1990, ApJ, 348, 412

Mikkola, S., \& Valtonen, M. J. 1992, MNRAS, 259, 115

Murphy, D. W, Browne, I. W. A., \& Perley, R. A. 1993, MNRAS, 264, 298

Nilsson, K. 1998, A\&AS, 132, 31

Novikov, I. D., \& Thorne, K. S. 1973, Black Hole Astrophysics, in Black Holes, ed. C. DeWitt, \& B. DeWitt (Gordon and Breach, New York)

Peters, P. C., \& Mathews, J. 1963, Phys. Rev., 131, 435

Quinlan, G. D., \& Hernquist, L. 1997, NewA, 2, 553

Rajagopal, M., \& Romani, R. W. 1995, ApJ, 446, 543

Readhead, A. C. S., Taylor, G. B., Xu, W., \& Pearson, T. J. 1996, ApJ, 460, 612

Rees, M. J., \& Saslaw, W. C. 1975, MNRAS, 171, 53

Roos, N. 1988, ApJ, 334, 95

Schweizer, F. 1982, ApJ, 252, 445

Shakura, N. I., \& Sunyaev, R. A. 1973, A\&A, 24, 337 
Shapiro, S. L., \& Teukolsky, S. A. 1983, Black Holes, White Dwarfs and Neutron Stars (A Wiley-Interscience Publication)

Smith, E. P., \& Heckman, T. M. 1989, ApJ, 341, 658 Smith, E. P. 1990, ApJ, 348, 38

Toomre, A., \& Toomre, J. 1972, ApJ, 178, 623

Valtaoja, E., \& Valtonen, M. J. 1984, A\&A, 130, 373

Valtaoja, E. 1986, Ap\&SS, 127, 103

Valtonen, M. J. 1996, Comments Astrophys., 18, No. 4, 191

Valtonen, M. J. 1996, MNRAS, 278, 186 (MV96)

Valtonen, M. J. 1999, ApJ, 520, 97

Valtonen, M. J., \& Heinämäki, P. 2000, ApJ, 530, 107

Valtonen, M. J., \& Kotilainen, J. 1989, AJ, 98, 117
Valtonen, M. J., Mikkola, S., Heinämäki, P., \& Valtonen, H. 1994, ApJS, 95, 69 (Paper I)

Valtonen, M. J., Saslaw, W. C., \& Aarseth, S. J. 1974, ApJ, 190, 258

Wilson, A. S., \& Colbert, E. J. M. 1995, ApJ, 438, 62

Woltjer, L. 1990, Active Galactic Nuclei, Saas-Fee Advanced Cource, ed. T. J.-L. Courvoisier, \& M. Mayor (SpringerVerlag)

Young, P. J., Westphal, J., Kristian, J., Wilson, C. P., \& Landauer, F. P. 1978, AJ, 221, 721

Young, P. J. 1976, AJ, 81, 807

Zirbel, E. L. 1996, ApJ, 476, 489

Zirbel, E. L. 1996, ApJ, 473, 713 